\title{
Presymptomatic genetic analysis during pregnancy for vascular type Ehlers-Danlos syndrome
}

This article was published in the following Dove Press journal:

International Medical Case Reports Journal

19 June 2014

Number of times this article has been viewed

\author{
Banyar Than Naing' \\ Atsushi Watanabe ${ }^{1,2}$ \\ Shinji Tanigaki ${ }^{3}$ \\ Masae Ono ${ }^{4}$ \\ Mitsutoshi Iwashita ${ }^{3}$ \\ Takashi Shimada ${ }^{1,2}$ \\ 'Department of Biochemistry and \\ Molecular Biology, Nippon Medical \\ School, Tokyo, Japan; ${ }^{2}$ Division of \\ Clinical Genetics, Nippon Medical \\ School Hospital, Tokyo, Japan; \\ ${ }^{3}$ Department of Obstetrics and \\ Gynecology, Kyorin University \\ School of Medicine, Tokyo, Japan; \\ ${ }^{4}$ Department of Pediatrics, Kyorin \\ University School of Medicine, \\ Tokyo, Japan
}

\begin{abstract}
The vascular type of Ehlers-Danlos syndrome (EDS), EDS type IV (Online Mendelian Inheritance in Man [MIM] \#130050) is characterized by thin, translucent skin, easy bruising, and arterial, intestinal, and/or uterine fragility during pregnancy, which may lead to sudden death. It is an autosomal dominant inherited disorder caused by type III procollagen gene (COL3A1: MIM \#120180) mutations. Approximately 50\% of the COL3A1 mutations are inherited from an affected parent, and $50 \%$ are de novo mutations. Each child of an affected individual has a $50 \%$ chance of inheriting the mutation and developing the disorder. Pregnant women with vascular EDS are at an increased risk of uterine and arterial rupture during the peripartum period, with high maternal morbidity and mortality rates. We report the first case of an asymptomatic 35-year-old woman at a risk of complications of vascular EDS who underwent presymptomatic evaluation during pregnancy. The sequencing results of both her brother and mother had a one-base-pair deletion, resulting in Glutamate at position 730 changing to Lysine and causing a frame shift and premature termination codon at 61 amino acids from the mutation position (p. Glu730Lysfs*61) on exon 32 of COL3A1. This deletion caused frameshift, leading to a premature termination codon (TAG) at 181 nucleotides downstream in exon 35 , which could not be detected by previous total RNA (ribonucleic acid) method. Thus, she was at risk of complications of vascular EDS, and diagnostic testing was employed at 8 weeks of pregnancy to minimize the risk of developing vascular EDS-related complications. The negative presymptomatic diagnostic result allowed the patient to choose normal delivery at term. Vascular EDS is a serious disorder, with high mortality, especially in high-risk women with vascular EDS during pregnancy. The presymptomatic genetic testing of vascular EDS during pregnancy for a high-risk family can help with the early establishment of preventive measures.
\end{abstract}

Keywords: vascular EDS, type III procollagen gene (COL3A1), testing

\section{Introduction}

The vascular type of Ehlers-Danlos syndrome (EDS), EDS type IV (Online Mendelian Inheritance in Man [MIM] \#130050) ${ }^{1,2}$ is an autosomal dominant inherited disorder. Its primary clinical features are rupture of the blood vessels or internal organs such as bowel and uterine rupture during pregnancy, which may lead to sudden death. . $^{2,3}$ Vascular EDS causes severe fragility of connective tissues, with arterial and intestinal ruptures and complications of surgical and radiological treatment, and is of particular importance to medical professionals of many specialties: surgeons, internists, radiologists, and obstetricians. An accurate diagnosis may help in the management of visceral complications. Vascular EDS is mainly caused by type III procollagen gene (COL3A1: MIM \#120180) mutations. ${ }^{4}$ The majority of identified mutations are point mutations that result in single amino acid substitutions for glycine in the GLY-X-Y
Correspondence: Atsushi Watanabe Division of Clinical Genetics, Nippon Medical School Hospital, I-I-5 Sendagi, Bunkyo-ku, Tokyo I I3-8603, Japan

Tel +8I 358146585

Email aw3703@nms.ac.jp 
repeat of the triple helical region. Approximately $50 \%$ of the COL $3 A 1$ mutations are inherited from an affected parent, and $50 \%$ are de novo mutations. ${ }^{5}$ Most individuals with vascular EDS will experience a significant medical problem in their lifetime, at rates of $25 \%$ and $80 \%$ by the ages of 20 years and 40 years, respectively. ${ }^{3}$

In some patients, potentially life-threatening complications of vascular EDS appear only during pregnancy, ${ }^{6}$ with the maternal mortality rate in the range of $12 \%-25 \%$ because of peripartum arterial or uterine rupture. ${ }^{3,7,8}$ To reduce the risks of late pregnancy and delivery, pregnant women with the COL3A1 mutation of vascular EDS are treated as high risk and selected for an early delivery by elective cesarean section. ${ }^{6,8,9}$ Genetic testing is one option that can help those in a family with the COL $3 A 1$ mutation to make informed decisions on risk management. Here, we report an asymptomatic woman at risk of complications of vascular EDS who underwent presymptomatic genetic testing during pregnancy.

\section{Case report}

An asymptomatic 35-year-old woman (client) at a risk of complications of vascular EDS during pregnancy came to our clinic for genetic counseling. The elder brother (proband) was diagnosed with vascular EDS with pneumothorax and COL3A1 heterozygous mutation, resulting in Glutamate at position 730 changing to Lysine and causing a frame shift and premature termination codon at 61 amino acids from the mutation position (p. Glu730Lysfs*61) by using the GenBank genetic sequence database available at http://www.ncbi.nlm. nih.gov/nuccore/NM_000090.3 (GenBank ID: NM_000090.3) as the reference, as shown in Figure 1. This deletion existed within the triple helical domain of COL $3 A 1$, but it caused a frameshift, resulting in a premature termination codon at 181 nucleotides downstream in exon 35 . This could not be detected by the previous total ribonucleic acid (RNA) method because of nonsense-mediated messenger RNA decay. Because there was no other family history of vascular EDS, we analyzed the COL3A1 mutation in both parents using polymerase chain reaction (PCR)-direct sequencing of the genomic deoxyribonucleic acid (DNA) to confirm whether or not the mutation occurred de novo. The mother had the same mutation, in spite of having no signs or symptoms of vascular EDS; therefore, the client had a $50 \%$ risk for the same mutation of COL $3 \mathrm{Al}$.

Before pregnancy, genetic counseling was performed for the client based on these results: she did not want to take the genetic test. However, when the client became pregnant, the obstetrician told her that it was important to consider pregnancy- and peripartum-related risks of vascular EDS and the need for an early delivery via elective cesarean section, even though the echocardiogram was normal, including the size of the main aorta. Thus, the client underwent genetic counseling again at 8 weeks of gestation and determined that she wanted to undergo presymptomatic genetic testing for vascular EDS. With the approval of the ethical committee of Nippon Medical School and after obtaining informed consent from the client, the presymptomatic genetic testing was conducted. ${ }^{10}$

To detect the mutation, PCR-direct sequencing using genomic DNA was performed. We used forward (5'CAGCATTCCATTCACCTAGG $3^{\prime}$ ) and reverse primers (5'TCGATTACTTTCCAGGAGTG $\left.3^{\prime}\right)$ that covered the
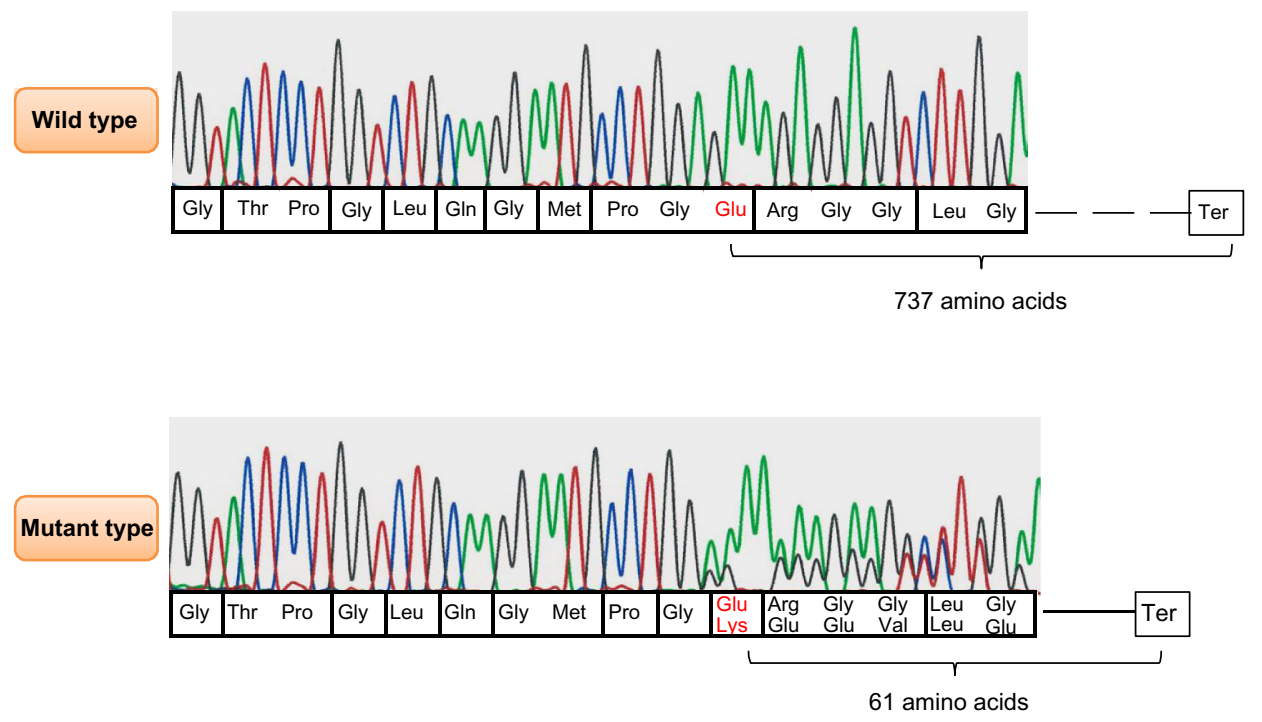

Figure I COL3A I results obtained by polymerase chain reaction direct sequencing method.

Notes: This mutation is a one-base pair deletion (c.2187delA) and because of the deletion, amino acid Glutamate at position 730 changed to Lysine causing frame shift and formation of premature termination codon at 61 amino acids from the mutation position (p.Glu730Lysfs*6I); the normal stop codon appeared after 737 amino acids. 
deletion position on exon 32. Capillary electrophoresis was performed using ABI Prism 3130 (Life Technologies, Carlsbad, CA, USA). We then undertook genotyping of common single nucleotide polymorphisms of COL $3 \mathrm{Al}$ around the mutation in each allele to confirm the mutation test in the family; these procedures have been previously reported. ${ }^{11}$ By combining the single nucleotide polymorphism genotyping results, we could prove which allele of each parent was derived to the proband and client (Figure 2). The client did not have the allele containing the COL3A1 mutation, allowing her to deliver normally at term. The healthy baby was born with no complications.

\section{Discussion}

Vascular EDS is usually caused by mutations of COL3A1 in single amino acid substitutions for glycine in the GLY-X-Y repeat of the triple helical region or in variant splice sites, causing a dominant-negative effect. ${ }^{12}$ Individuals with these mutations have severe symptoms because of high penetrance. COL3A1 haploinsufficiency, which has been found to be an alternative mechanism, ${ }^{13}$ results in a variety of vascular EDS with delayed onset of complications and longer life expectancy compared with other mutation types. ${ }^{14}$ The mother with a COL3A1 mutation in our report did not show any clinical symptoms, possibly because of haploinsufficiency caused by nonsense-mediated messenger RNA decay. Some vascular EDS patients with null mutations including

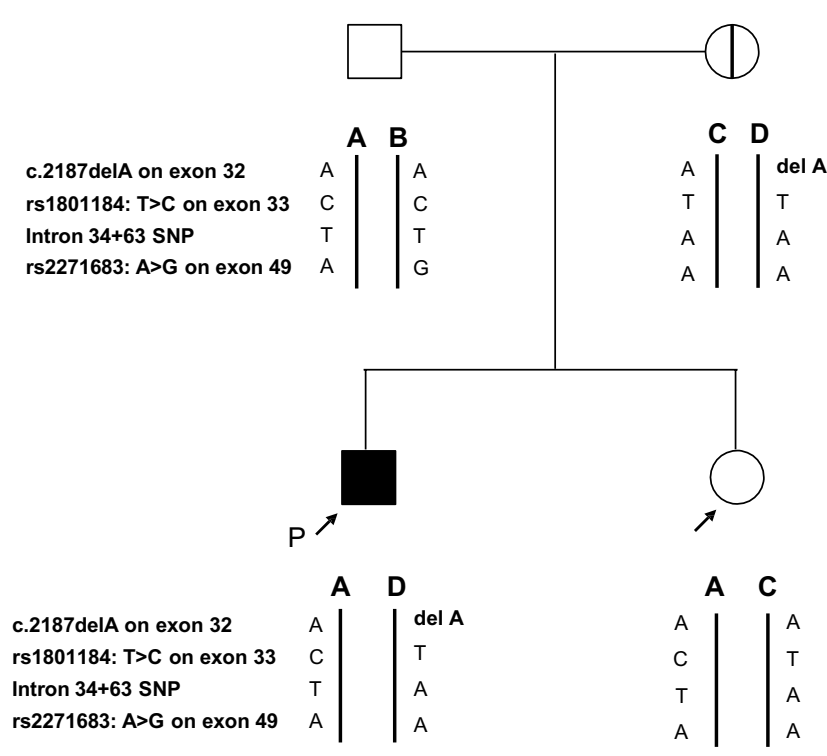

Figure 2 The family pedigree with the result of the allele confirmation test. Notes: The proband had a one-base-pair deletion (c.2187delA) on exon 32. The mother had the same mutation but no signs or symptoms of vascular Ehlers-Danlos syndrome. Using the informative SNPs ( $r$ I80II84, intron 34+63 and rs2271683), we determined from which parent each allele was derived (for the proband and patient). This clearly proved the familial relationship of the members.

Abbreviations: P, proband; SNP, single nucleotide polymorphism. nonsense-mediated messenger RNA decay also showed the same severe phenotypes, like that of patients with missense or splice-site mutations. ${ }^{14}$ Severities of vascular EDS were varied between the members of the family with the same mutation. In vascular EDS, genotype-phenotype correlations also add important information in predicting outcome and selecting the treatment. Determining the mutation type is important for the management of vascular EDS. Although de novo COL $3 A 1$ mutations occur in approximately $50 \%$ of cases, genetic testing of parents is important for evaluating the risk in other family members.

Management is also necessary for vascular EDS following presymptomatic diagnosis. In pregnant women with vascular EDS and COL $3 A 1$ mutation, it is prudent to follow the high-risk obstetric management protocols. ${ }^{6,9}$ It is essential to educate pregnant women with regard to the possible complications, and the requirement for close monitoring is recommended. ${ }^{7,15,16}$ During pregnancy, these patients are more susceptible to complications such as uterine and vascular rupture because of plasma volume expansion. ${ }^{17}$ Depending on the patient's condition, one of the choices is to choose early delivery via elective cesarean section ( 32 weeks' gestation) because the risk of complication is considered greatest during labor, delivery, and immediately postpartum. ${ }^{18}$ In order to be prepared in an emergency, from a surgical perspective, we also need to be aware that vessels are often very friable. This observation was made mainly during operations. ${ }^{19}$ Thus, a firm diagnosis of vascular EDS may help in the management of visceral complications, pregnancy, and genetic counseling. ${ }^{12}$

To the best of our knowledge, this is the first report on presymptomatic genetic testing in a pregnant woman with a family history of vascular EDS and known COL3A1 mutation. Presymptomatic genetic testing is an important choice for individuals at risk of complications of vascular EDS during pregnancy and facilitates informed decisionmaking and effective management of complications.

\section{Acknowledgments}

The authors thank the patient and her family members who participated in this study. This work was supported in part by a grant from Grants-in Aid for Scientific Research from the Ministry of Health, Education, Culture, Sports, Science, and Technology of Japan.

\section{Disclosure}

The authors report no conflicts of interest in this work.

\section{References}

1. Germain DP. Clinical and genetic features of vascular Ehlers-Danlos syndrome. Ann Vasc Surg. 2002;16(3):391-397. 
2. Watanabe A, Kosho T, Wada T, et al. Genetic aspects of the vascular type of Ehlers-Danlos syndrome (vEDS, EDSIV) in Japan. Circ J. 2007;71(2):261-265.

3. Pepin M, Schwarze U, Superti-Furga A, et al. Clinical and genetic features of Ehlers-Danlos syndrome type IV, the vascular type. $N$ Engl J Med. 2000;342(10):673-680.

4. Dalgleish R. The Human Collagen Mutation Database 1998. Nucleic Acids Res. 1998;26(1):253-255.

5. Germain DP. Ehlers-Danlos syndrome type IV. Orphanet J Rare Dis. 2007;2:32

6. Hammond R, Oligbo N. Ehlers-Danlos syndrome type IV and pregnancy. Arch Gynecol Obstet. 2012;285(1):51-54.

7. Lurie S, Manor M, Hagay ZJ. The threat of type IV Ehlers-Danlos syndrome on maternal well-being during pregnancy: early delivery may make the difference. J Obstet Gynaecol. 1998;18(3):245-248.

8. Rudd NL, Nimrod C, Holbrook KA, et al. Pregnancy complications in type IV Ehlers-Danlos Syndrome. Lancet. 1983;1(8314-8315):50-53.

9. Peaceman AM, Cruikshank DP. Ehlers-Danlos syndrome and pregnancy: association of type IV disease with maternal death. Obstet Gynecol. 1987;69(3 Pt 2):428-431.

10. Skirton H, Goldsmith L, Jackson L, et al. Quality in genetic counselling for presymptomatic testing - clinical guidelines for practice across the range of genetic conditions. Eur J Hum Genet. 2013;21(3):256-260.

11. Naing BT, Watanabe A, Shimada T. A novel mutation screening system for Ehlers-Danlos syndrome, vascular type by high-resolution melting curve analysis in combination with small amplicon genotyping using genomic DNA. Biochem Biophys Res Commun. 2011;405(3): $368-372$.
12. Watanabe A, Shimada T. Vascular type of Ehlers-Danlos syndrome. J Nippon Med Sch. 2008;75(5):254-261.

13. Schwarze U, Schievink WI, Petty E, et al. Haploinsufficiency for one COL3A1 allele of type III procollagen results in a phenotype similar to the vascular form of Ehlers-Danlos syndrome, Ehlers-Danlos syndrome type IV. Am J Hum Genet. 2001;69(5):989-1001.

14. Leistritz DF, Pepin MG, Schwarze U, et al. COL3A1 haploinsufficiency results in a variety of Ehlers-Danlos syndrome type IV with delayed onset of complications and longer life expectancy. Genet Med. 2011;13(8):717-722.

15. Chetty SP, Shaffer BL, Norton ME. Management of pregnancy in women with genetic disorders, Part 1: Disorders of the connective tissue, muscle, vascular, and skeletal systems. Obstet Gynecol Surv. 2011;66(11):699-709.

16. Bergqvist D, Bjorck M, Wanhainen A. Treatment of vascular EhlersDanlos syndrome: a systematic review. Ann Surg. 2013;258(2): 257-261.

17. Palmquist M, Pappas JG, Petrikovsky B, et al. Successful pregnancy outcome in Ehlers-Danlos syndrome, vascular type. J Matern Fetal Neonatal Med. 2009;22(10):924-927.

18. ErezY, Ezra Y, Rojansky N. Ehlers-Danlos type IV in pregnancy. A case report and a literature review. Fetal Diagn Ther. 2008;23(1):7-9.

19. Kimura K, Sakai-Kimura M, Takahashi R, et al. Too friable to treat? Lancet. 2010;375(9725):1578.
International Medical Case Reports Journal

\section{Publish your work in this journal}

The International Medical Case Reports Journal is an international, peer-reviewed open-access journal publishing original case reports from all medical specialties. Previously unpublished medical posters are also accepted relating to any area of clinical or preclinical science. Submissions should not normally exceed 2,000 words or

\section{Dovepress}

4 published pages including figures, diagrams and references. The manuscript management system is completely online and includes a very quick and fair peer-review system, which is all easy to use. Visit http://www.dovepress.com/testimonials.php to read real quotes from published authors. 